\title{
Iron (III) Porphyrin Supported on S and N co-Doped Graphene Quantum Dot as an Efficient Photocatalyst for Aerobic Oxidation of Alcohols under Visible Light Irradiation
}

\author{
Mojtaba Mahyari $^{* a}$, Yasamin Bide $^{\mathrm{b}}$, Jaber Nasrollah Gavgani ${ }^{\mathrm{c}}$

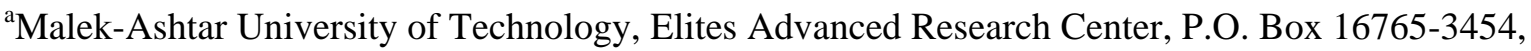 \\ Tehran, Iran \\ ${ }^{\mathrm{b}}$ Faculty of Chemistry, Shahid Beheshti University, G. C., P. O. Box 19396-4716, Tehran, Iran \\ ${ }^{c}$ Department of Polymer Engineering and Color Technology, Amirkabir University of Technology, \\ P.O.Box 15875-4413, Tehran, Iran
}

\begin{abstract}
In this study, we report the synthesis and impressive performance of Fe(III)tetra(4sulfonatophenyl) porphyrins (FeTSPP) supported on S and N co-doped graphene quantum dots (S, N: GQDs) as efficient, recyclable, and noble metal free heterogeneous photocatalyst for the selective aerobic oxidation of alcohols to the corresponding carbonyl compounds. It was found that FeTSPP@S, N: GQDs photocatalyst represents a novel chemical function for efficient visible light catalyzed aerobic alcohol oxidation with good conversions and selectivity in water as a green solvent at room temperature. We envision that FeTSPP@S, N: GQDs provide a promising candidate for the development of highly efficient, recyclable, and industrially applicable photocatalyst in fine chemical production with high activity.
\end{abstract}

Keywords: Graphene quantum dots, Photocatalyst, Aerobic oxidation, Iron (III) porphyrin.

*Email: m_mahyari@sbu.ac.ir

Tel:+982122799167 


\section{Introduction}

Oxidation of organic compounds especially selective oxidation of alcohols to corresponding carbonyl compounds are one of the most important organic reactions in synthetic organic chemistry and fine chemicals industry [1-4]. According to the principles of green chemistry and sustainable design from the both environmental and economic viewpoints, development of efficient and environmentally benign heterogeneous processes with alternative green energies, and lowest-level waste, as well as stability in reaction media are the priorities [2, 5, 6]. Although there are several traditional chemical processes for functional group transformations [7-15] which are contradictory to the principles of green chemistry, nowadays, catalytic aerobic oxidation represents one of the most environmentally efficient technologies in chemical industry. The main oxidizing agent in this route is molecular oxygen, but the preparation of active oxygen at ambient temperature and pressure for selective oxidation reaction is one of the challenging issues. The visible light photocatalysis is the promising and environmentally approach for the catalytic activation of $\mathrm{O}_{2}[16-19]$.

Metalloporphyrins as the well-known biomimetic photocatalysts using successfully as models for the cytochrome P-450 enzyme [20], have been widely investigated in variety of oxidation reactions [21-24]. Additionally, the metalloporphyrin derivatives, owning to their large $\pi$ electron systems leading to high absorption in visible light region [16, 25], were used as a dye sensitizer in the photocatalysis processes. However, the metalloporphyrins as homogeneous catalysts have a number of weaknesses, including difficult recovery and reusing, intermolecular self-oxidation, dimerization or decomposition during the reaction, high cost of preparation and easily deactivation which can overcome by their immobilization on solid surface [26-29]. The carbon materials as new type of solid supports for heterogeneous catalysis not only offer some unique features, but also play a leading role for the enhancement of catalytic performance of supported metalloporphyrins [30, 31].

Graphene quantum dots (GQDs) with narrow size distribution, high mobility and tunable band gap have attracted considerable attention for various applications such as light emitting diodes, bioimaging, optoelectronic devices, and especially photocatalysis [32-34]. As a consequence, tremendous attraction has been paid to the development of GQDs as photogenerated carriers because of their remarkable properties including large surface area, excellent electronic and 
optical properties, availability, low cytotoxicity, biocompatibility, unique photostability, excellent dispersibility in water, and high loading capacity [34-38]. Most of the developed methods in the preparation of carbon-based dots are unsatisfactory owning to low yields, low fluorescent quantum yield, complicated procedures and also need to expensive equipment [3942]. Most recently, nitrogen and sulfur co-doped GQDs (S, N: GQDs) were proposed as highly fluorescent dots [32, 43]. Moreover, its unique optic and electronic properties as well as broad absorption bands in the visible region would be a great advantage for broad applications of S, N: GQDs.

Because of the above mentioned reasons and also our interest in the synthesis of green and new catalysts [31, 44-50], herein for the first time, we report the successful synthesis and excellent catalytic activity of $\mathrm{Fe}$ (III)tetra(4-sulfonatophenyl) porphyrins (FeTSPP) supported on S, N: GQDs as novel visible light photocatalyst with unique properties for the selective aerobic oxidation of alcohols to the corresponding carbonyl groups. The significantly enhanced conversions, mild reaction conditions, good recyclability and being environmentally-friendly made this photocatalyst to provide a novel platform for the selective oxidation of organic compounds in industry.

\section{Experimental}

\subsection{Materials}

Citric acid (+ 99\%), urea, and thiourea were purchased from Sigma Aldrich Co. Fe(III)tetra(4sulfonatophenyl)porphyrins were obtained from Merck Co. Other reagents were of analytical grade and used as received from Sigma Aldrich Co. Doubly distilled water was used throughout the experiments.

\subsection{Synthesis of $\mathbf{N}$ doped GQDs (N: GQDs)}

Citric acid $(0.21 \mathrm{~g}, 1 \mathrm{mmol})$ and urea $(0.18 \mathrm{~g}, 3 \mathrm{mmol})$ were dissolved into $5 \mathrm{~mL}$ water, and stirred to form a clear solution and then it was transferred into a $20 \mathrm{ml}$ Teflon lined stainless autoclave. The sealed autoclave was heated to $160{ }^{\circ} \mathrm{C}$ for 4 hours. Finally, N: GQDs were collected by adding ethanol into the solution and centrifuged at $5000 \mathrm{rpm}$ for $5 \mathrm{~min}$. The $\mathrm{N}$ : GQDs powder can be easily redispersed into water [32, 51].

\subsection{Synthesis of $S, N$ co-doped GQDs (S, N: GQDs)}


In our previous work [52], S, N: GQDs were synthesized as follows: citric acid (0.21 g, $1 \mathrm{mmol})$ and thiourea $(0.23 \mathrm{~g}, 3 \mathrm{mmol})$ were dissolved in $5 \mathrm{~mL}$ water, and stirred to form a clear solution. Then the solution was transferred into a $20 \mathrm{~mL}$ Teflon lined stainless autoclave and heated at 160 ${ }^{\circ} \mathrm{C}$ for 4 hours. The final product was collected by adding ethanol into the solution and centrifuged at $5000 \mathrm{rpm}$ for $10 \mathrm{~min}$. The obtained solid can be easily redispersed into water [32, $43,51,52]$.

\subsection{Synthesis of $\mathrm{Fe}($ III)tetra(4-sulfonatophenyl) porphyrins supported on $\mathrm{S}$, $\mathrm{N}$ co-doped graphene quantum dots (FeTSPP@S, N: GQDs)}

$\mathrm{S}, \mathrm{N}$ : GQDs $(0.5 \mathrm{~g})$ were sonicated in $20.0 \mathrm{~mL}$ water for $30 \mathrm{~min}$ to form a clear solution. Then FeTSPP (0.1 g) was slowly added under mechanical stirring and the mixture solution was heated to $100{ }^{\circ} \mathrm{C}$ for $24 \mathrm{~h}$. The unsupported S, N: GQDs were removed via Soxhlet extraction with water and the final product was obtained $[48,53]$. The synthesis of FeTSPP@S, N: GQDs is schematically shown in Fig. 1.

\subsection{General procedure for the photocatalytic aerobic oxidation of alcohols}

Photocatalytic tests were performed by stirring the mixture solutions of (1.0 mmol) alcohol, (1 mg) FeTSPP@S, N: GQDs, and (5.0 mL) water in open air at room temperature for at least 10 min before irradiation. The samples were placed on narrow glass tubes $(10.0 \mathrm{~mL}$ capacity) with magnetic stirring bar. The test tubes were put in a water bath containing visible light source [fluorescent circular lamp, $22 \mathrm{~W}, 230 \mathrm{~V}, 32400 \mathrm{LUX}$, intensity determined with light meter (LT lutron model YK-25001x) and $\lambda>350 \mathrm{~nm}$ ] or sunlight (62000 LUX) and irradiated for the required reaction time. The course of the irradiation was followed by taking one test tube at the required reaction time and analyzing the products retained in the solid. The solids were extracted by redispersing the powder in $3 \mathrm{ml}$ of dichloromethane. The combined extracts (supernatant plus products recovered from the solid) were analyzed by Gas chromatography and spectral changes were monitored with Shimadzu UV-2100. GC conversions were obtained based on the amount of remaining alcohol in the reaction mixture relative to the standard sample containing $1.0 \mathrm{mmol}$ alcohol.

\subsection{Characterization}


Infrared spectra were recorded on a Bomem MB-Series Fourier transform infrared (FTIR) spectrophotometer. X-ray photoelectron spectroscopy (XPS) analysis was performed using a VG multilab 2000 spectrometer (ThermoVG scientific) in an ultra-high vacuum. High resolution transmission electron microscopy (HRTEM) images were recorded on a HRTEM JEOL 2100 system operating at $200 \mathrm{kV}$. The specimens were prepared by drop-casting the sample solution onto a carbon coated copper grid, followed by drying at room temperature. Gas chromatography (GC) was carried out on a Varian model 3600 gas chromatograph (Varian Iberica, Madrid, Spain) equipped with a split/splitless capillary injection port and flame ionization detector (FID). A CP-Sil-8 fused silica capillary column $(25 \mathrm{~m} \times 0.32 \mathrm{~mm}$ i.d. and $0.52 \mathrm{~mm}$ film thickness $)$ was employed from Chrompack. GC conversions and reaction yields were obtained using n-decane as an internal standard. TOC analyses were carried out by using a 5000 A Shimadzu TOC analyser. The Carbon balance was reported by the value obtained by TOC determination. ${ }^{1} \mathrm{H}-$ NMR Spectra were recorded on a Bruker DRX-300 Avance spectrometer $300.13 \mathrm{MHz}$; chemical shifts $\left(\delta\right.$ scale) are reported in parts per million $(\mathrm{ppm}) .{ }^{1} \mathrm{H}-\mathrm{NMR}$ Spectra are reported in order: number of protons, multiplicity and approximate coupling constant ( $\mathrm{J}$ value) in hertz $(\mathrm{Hz})$; signals were characterized as s (singlet), d (doublet), t (triplet), m (multiplet), br s (broad signal) and $\mathrm{Ar}$ (aryl). The ${ }^{13} \mathrm{C}-\mathrm{NMR}$ spectra were recorded at $75.47 \mathrm{MHz}$; chemical shifts ( $\delta$ scale) are reported in parts per million (ppm). $\mathrm{CDCl}_{3}$ was used as a solvent in ${ }^{1} \mathrm{H}-,{ }^{13} \mathrm{C}-\mathrm{NMR}$ analyses. Ultrasonic bath (EUROSONIC® 4D ultrasound cleaner with a frequency of $50 \mathrm{kHz}$ and an output power of $350 \mathrm{~W}$ ) was used to disperse materials in solvent. X-ray powder diffraction (XRD) data were collected on an XD-3A diffractmeter using $\mathrm{Cu} \mathrm{K} \alpha$ radiation by using silicon as a standard material. The interlayer spacing (d) was determined according to Bragg's law: 2 dsin $\theta$ $=\mathrm{n} \lambda$, where $\mathrm{n}$ is an integer determined by the given order, and $\lambda$ is the wavelength. UltravioletVisible (UV-Vis) spectra were obtained using a Shimadzu UV-2100 spectrophotometer. Thermogravimetric analysis (TGA) was carried out using a thermoanalyzer instrument (Mettler Toledo, SDTA 851) at a linear heating rate of $10{ }^{\circ} \mathrm{C} / \mathrm{min}$ under a nitrogen flow. AA-680 Shimadzu (Kyoto, Japan) flame atomic absorption spectrometer (AAS) with a deuterium background corrector was used for determination of the metal.

\section{Results and discussion}

\subsection{Characterization of FeTSPP@S, N: GQDs photocatalyst}


The un-doped GQDs show low photoluminescense (quantum yield) compared to S, N: GQDs, due to localization of electron-hole pairs and reduced band gap, suggesting relatively strong electron affinity of $\mathrm{S}$ and $\mathrm{N}$ atoms [32]. Moreover, previous experimental observations and quantum-mechanical calculations have proved the strong electron-withdrawing ability of the $S$ and $\mathrm{N}$ atoms within the conjugated $\mathrm{C}$ plane based on the typical $\pi-\pi^{*}$ stacking transition of aromatic $\mathrm{sp}^{2}$ domains [54-56]. These results demonstrate that the as-prepared S, N: GQDs not only possess unique optical and electronic properties, but also represent broad absorption bands in the visible region owning to the $\mathrm{S}$, N-doping-induced modulation of the chemical and electronic characteristics of the GQDs $[32,57]$. Therefore, S, N: GQDs as photocatalytic carriers could improve the use of solar light for energy conversion in aerobic oxidation reaction because of their broad visible light absorption [32, 43]. In addition, S, N: GQDs can be used as support for photocatalytic aerobic oxidation reactions under visible light irradiation. On the other hand, FeTSPP was used as active biomimetic site [31, 58-61] on S, N: GQDs surface leading to good biomimetic photocatalytic efficiency. It can be mentioned that FeTSPP has unique photosensitivity which can activate triplet oxygen through excitation into singlet oxygen under proper lighting [16]. As we mentioned earlier, the weaknesses of FeTSPP as a heterogeneous catalyst can be overcome by its immobilization on $\mathrm{S}, \mathrm{N}$ : GQDs surface. Additionally, the introduction of FeTSPP to S, N: GQDs surface leads to enhance the hydrophilic properties of the photocatalyst by ionic interaction, and consequently improves the dispersity and stability of the photocatalyst in aqueous solution. Moreover, the effective contact area and surface charge of FeTSPP were enhanced due to the excellent electron conductivity and large surface area of the S, N: GQDs. The non-covalent bonding between S, N: GQDs and FeTSPP can undergo fast transfer of electron which causes a large red shift of absorption wavelength [62]. It is important to mention that good interaction between oxidizing agent and reagent on photocatalyst surface has been considered in the design of as-prepared FeTSPP@S, N: GQDs. Therefore, to improve the chemical interaction of catalyst surface, some attempts have been made such as transition metals loading and introduction of dopants such sulfur and nitrogen [30, 63]. In addition, oxygen molecules were activated by their interaction with $\mathrm{N}$-doped support leading to oxygen adsorption on the bridge sites of graphene with migration energy of over $1.0 \mathrm{eV}$ [64]. Besides, the presence of nitrogen dopant on the surface of support is necessary to make ionic interaction between FeTSPP and S, N: GQDs and also easy functionalization of support with FeTSPP [48]. 
TEM image (Fig. 2) shows that the size of the as-prepared FeTSPP@S, N: GQDs is distributed in the range from 2 to $4 \mathrm{~nm}$, with mean diameters of $2.81 \pm 0.35 \mathrm{~nm}$, which are exactly corresponded with those of GQDs synthesized electrochemically, but smaller than those of Nfree GQDs prepared hydrothermally [65, 66]. A representative HRTEM image (inset of Fig. 2) indicates a lattice spacing distance of $0.35 \mathrm{~nm}$ which is similar to that of graphite (002) facet. The above results reveal the high crystallinity of FeTSPP@S, N: GQDs which might be composed of nanocrystalline cores of graphitic $\mathrm{sp}^{2}$ carbon atoms. This result is similar to that of many other reported S, N: GQDs [43, 67-69]. Based on our previous work [52], Raman spectrum, XRD profile and UV-Vis absorption spectrum of S, N: GQDs have been shown in Fig. S1. Raman spectrum of S, N: GQDs exhibits two peaks at $1372 \mathrm{~cm}^{-1}$ (D band) and $1579 \mathrm{~cm}^{-1}$ (G band) ascribed to the planar configuration of $\mathrm{sp}^{2}$ bonded carbon and structural disorder, respectively (Fig. S1a) [52]. In comparison with original graphene film, the S, N: GQDs show a broader $\mathrm{D}$ band, indicating that the intercalation of $\mathrm{S}$ and $\mathrm{N}$ atoms into the graphene layers (carbon backbone) breaks the translational symmetry (disordered structures) [54]. Figure S1b shows XRD profile of S, N: GQDs [52]. The as-prepared S, N: GQDs exhibited a distinct peak at $2 \theta=24.6^{\circ}$ which is substantially higher than that of the graphene film (ca. $23.5^{\circ}$ ). Therefore, the interlayer spacing in the $\mathrm{S}, \mathrm{N}$ : GQDs (ca. $0.35 \mathrm{~nm}$ ) is more compact than the original graphene film (ca. $37 \mathrm{~nm}$ ) as determined by Bragg's law. The reduced interlayer spacing in S, N: GQDs compared to the original graphene film could be ascribed to the possible formation of hydrogen bonding between oxygen containing functional groups on the surface of the tiny graphene sheets in S, N: GQDs, leading to effective $\pi-\pi$ stacking of graphene layers [32]. The UV-Vis absorption spectrum of the as-prepared S, N: GQDs has been presented in Fig. S1c [52]. The absorption peak at $337 \mathrm{~nm}$ is attributed to $\mathrm{n}->\pi^{*}$ transition of the $\mathrm{C}=\mathrm{O}$ bond, and the absorption bands at 422,550 , and $595 \mathrm{~nm}$ may be ascribed to $\pi->\pi^{*}$ and $\mathrm{n}->\pi^{*}$ of $\mathrm{C}=\mathrm{S}$ and $\mathrm{S}=\mathrm{O}$, respectively [32, $34,38,67,68,70]$. These results are consistent with the color change of product from a green solution (under normal light) to light blue when excited with $360 \mathrm{~nm}$ UV beam (see inset Fig. S1c).

FTIR spectrum of FeTSPP@S, N: GQDs has been shown in Fig. 3 displaying the absorption bands at 3000-3500 $\mathrm{cm}^{-1}(v \mathrm{O}-\mathrm{H}, \mathrm{N}-\mathrm{H}), 1709 \mathrm{~cm}^{-1}\left(v \mathrm{C}=\mathrm{O}\right.$, carboxylic), and $1667 \mathrm{~cm}^{-1}(v \mathrm{C}=\mathrm{O}$, amide). Also the bands at 1612 and $1401 \mathrm{~cm}^{-1}$ are assigned to the bending vibrations of $\mathrm{C}=\mathrm{C}$ and $\mathrm{C}-\mathrm{N}$, respectively. The peaks at $1083 \mathrm{~cm}^{-1}$ and $635 \mathrm{~cm}^{-1}$ are attributed to the stretching vibrations 
of $\mathrm{C}=\mathrm{S}$ and $\mathrm{C}-\mathrm{S}$, respectively [32]. The results confirmed that GQDs are successfully doped with $\mathrm{S}$ and $\mathrm{N}$ with thiourea $[32,52]$. Moreover, the bands at $1585-1505 \mathrm{~cm}^{-1}$ range are attributed to asymmetric and symmetric deformation of protonated imine $\left(=\mathrm{NH}^{+}\right)[71,72]$. The two bands at $1015 \mathrm{~cm}^{-1}$ and $1150 \mathrm{~cm}^{-1}$ are ascribed to the symmetric and asymmetric stretching vibrations of sulfonate anion $\left(-\mathrm{SO}_{3}{ }^{-}\right)$groups [73-75]. In this case, proton transfer from FeTSPPs to the $\mathrm{S}, \mathrm{N}$ : GQDs surface, leading to strong Coulombic interactions between the sulfonate anion and imine cation $[48,72,76,77]$. This would be suggested that the FeTSPPs are supported on the S, N: GQDs surface by ionic interaction (See Fig. 1).

XPS measurement was performed to determine the composition of the as-prepared FeTSPP@S, N: GQDs photocatalyst. The XPS spectrum of FeTSPP@S, N: GQDs indicates five peaks at 533, 400, 284, 227, and $164 \mathrm{eV}$ which are attributed to $\mathrm{O} 1 \mathrm{~s}, \mathrm{~N} \mathrm{1s}, \mathrm{C} 1 \mathrm{~s}, \mathrm{~S} 1 \mathrm{~s}$ and $\mathrm{S} \mathrm{2p}$, respectively (Fig. 4a) [32, 43, 52, 67]. Moreover, additional peaks at 711.6 and $724.4 \mathrm{eV}$ are assigned to $\mathrm{Fe} 2 \mathrm{p}_{3 / 2}$ and $\mathrm{Fe} 2 \mathrm{p}_{1 / 2}$, respectively (inset of Fig. 4a) $[75,78]$. The high-resolution $\mathrm{N} 1 \mathrm{~s}$ spectrum of FeTSPP@S, N: GQDs presents three peaks at 398.5, 400.8, and 402.2eV, which are assigned to imine $\left(=\mathrm{N}^{-}\right)$, amine $(-\mathrm{NH}-)$, and protonated imine $\left(=\mathrm{NH}^{+}\right)$, respectively (Fig. $\left.4 \mathrm{~b}\right)[43$, 77, 79]. The high resolution S2p spectrum of FeTSPP@S, N: GQDs obviously reveals three peaks at 163.1, 164.3, and $168.3 \mathrm{eV}$, which are attributed to $S 2 \mathrm{p} 3 / 2$ and $S 2 \mathrm{p} 1 / 2$ of thiophene, $\mathrm{S}=\mathrm{O}$ bonding, and sulfur within ring-attached mono-substituents of $-\mathrm{SO}_{3}{ }^{-}$or $-\mathrm{SO}_{3} \mathrm{H}$ group, respectively, which are consistent with FTIR results (Fig. 3) [66, 77, 80, 81]. These results indicate the successful doping of graphene of S, N: GQDs with $\mathrm{N}$ and $\mathrm{S}$ and also exhibit that FeTSPP is supported on S, N: GQDs surface by ionic interaction between sulfonate anion and imine cation. Based on the above results and previous reports [32, 51, 82], we proposed that at initial stage of the reaction, citric acid self-assembled into nanosheets structure owning to the inter-molecular H-bonding. Then, dehydrolysis process happened and graphene nanoparticles formed with lots of carboxyl and hydroxyl groups under hydrothermal condition. Finally, $\mathrm{NH}_{2}$ and $\mathrm{S}$ groups of thiourea reacted with carboxyl and hydroxyl groups to form $\mathrm{S}, \mathrm{N}$ co doped GQDs.

The thermal behavior of S, N: GQDs and FeTSPP@S, N: GQDs are shown in Fig. 5. In the case of S, N: GQDs, the weight loss of about $5 \%$ at $70-100{ }^{\circ} \mathrm{C}$ is attributed to the loss of water. The second weight loss of $\sim 15 \%$ at $310-400{ }^{\circ} \mathrm{C}$ is probably related to the decomposition of doped sulfonic and imine groups. 


\subsection{Choice of reaction conditions}

To investigate the photocatalytic properties of as-prepared FeTSPP@S, N: GQDs photocatalyst, aerobic oxidation reaction of benzyl alcohol was performed as a model reaction. Our results showed that the yield and selectivity were remarkably affected by photocatalyst loading. The desired yield and selectivity were achieved by using $0.1 \mathrm{~mol} \% \mathrm{Fe}(1 \mathrm{mg})$ with respect to benzyl alcohol (Table 1, entries 1-4). The evaluation of three solvents, water, acetonitrile, and dichloromethane demonstrated that water significantly enhanced the yield and selectivity of benzyl alcohol oxidation. The possibility of using water as a green reaction medium is a significant advantage because of its ionic interaction with as-prepared photocatalyst and very low solubility of the organic compounds in water, providing easy separation of the final products (Table 1, entries 4 -7).

Table. 1 Optimization of the reaction conditions for aerobic oxidation reaction of benzyl alcohol

\begin{tabular}{|c|c|c|c|c|}
\hline Entry & $\begin{array}{c}\text { Amount of } \\
\text { photocatalyst } \\
(\mathrm{Fe} \text { (III) content } / \mathrm{mol} \\
\% \text { ) }\end{array}$ & Solvent & Conversion (\%) & Selectivity (\%) \\
\hline 1 & None & $\mathrm{H}_{2} \mathrm{O}$ & 5 & 15 \\
\hline 2 & 0.02 & $\mathrm{H}_{2} \mathrm{O}$ & 37 & 57 \\
\hline 3 & 0.05 & $\mathrm{H}_{2} \mathrm{O}$ & 65 & 75 \\
\hline 4 & 0.10 & $\mathrm{H}_{2} \mathrm{O}$ & 88 & 87 \\
\hline 5 & 0.10 & $\mathrm{Solvent} \mathrm{free}$ & 50 & 95 \\
\hline 6 & 0.10 & $\mathrm{CH}_{3} \mathrm{CN}$ & 36 & 76 \\
\hline 7 & 0.10 & $\mathrm{CH}_{2} \mathrm{Cl}$ & 25 & 80 \\
\hline
\end{tabular}

\subsection{Conversion efficiency}

A variety of primary aromatic alcohols were applied for the aerobic oxidation reaction under air at room temperature. The conversions, selectivity and carbon balances of different reagents are 
reported in Fig. 6. Under these optimum conditions, all reagents completely converted to the related hydrocarbons with high yields and selectivity. In order to check the potentiality of this process, the as obtained products including benzaldehyde, 4-methoxybenzaldehyde, 4bromobenzaldehyde, 4-chlorobenzaldehyde were separated and analysed. ${ }^{1} \mathrm{H}-\mathrm{NMR}$ and ${ }^{13} \mathrm{C}-\mathrm{NMR}$ analyses (Table S1) confirmed the high purity of the products (> 99\%). It is important to investigate the effect of the light sources on the conversion of aerobic oxidation reactions. Encouraged by the promising results was shown in Fig. 6, the conversion of alcohols under photocatalytic conditions was closely corresponded with its of obtaining under white light source. It is obvious that these reactions can proceed under solar radiation, since the spectral distribution curve of a fluorescent lamp is similar to that of sunlight. Therefore, solar radiation, in accordance with the principles of "green chemistry", can be selected as an infinite source of clean energy in industrial plans.

The leaching of active sites (metal species) into the reaction medium is a very important subject to be investigated when heterogeneous catalysts are used. In order to study the leaching, a series of tests were carried out as described below. Preliminary survey showed that FeTSPP@S, N: GQDs photocatalyst is very stable to air and moisture. Then, in a separate test, the photocatalyst was filtered off after $50 \%$ conversion under the optimized conditions while the reaction is allowed to continue. The obtained results showed that the reaction did not proceed significantly without photocatalyst. The yield of reaction obtained by GC was 5\%. In addition, the AAS results of the filtrate also proved that the Fe (III) content in the reaction solution was below the detection limit ( $0.1 \mathrm{ppm})$. By repeating this experiment for FeTSPP@S, N: GQDs photocatalyst, the leaching content was achieved to be $0.11 \mathrm{ppm}$. As a consequence, we conclude that as expected, the metal leaching was inhibited owning to the high affinity of Fe(III) and the S, N: GQDs support.

The aerobic photocatalytic oxidation reactions of benzyl alcohol were studied using FeTSPP@S, N: GQDs, S, N: GQDs, N: GQDs, GQDs, and FeTSPP under the optimized conditions (Entries 1-5, Table 2). It is worth mentioning that the metal contents of FeTSPP@S, N: GQDs and FeTSPP have been considered as equals to have a correct comparison. Preliminary experiments were performed with $5 \mathrm{mg}$ of GQDs, N: GQDs, S, N: GQDs under optimized conditions, which did not exhibit good conversion (Table 2, entry 2-4). However, when a small amounts of pure 
FeTSPP and FeTSPP@S, N: GQDs were added to the reaction mixture, the conversion was enhanced significantly in the presence of FeTSPP@S, N: GQDs, and benzyl aldehyde was produced in 56 and $85 \%$ yield, respectively. It has been suggested that S, N: GQDs as support can enhance the adsorption of aromatic reagents owning to the $\pi-\pi$ bonding between benzene skeleton of the reagents and GQDs. As we mentioned earlier, S, N: GQDs promote oxygen adsorption on the bridge sites of GQDs, leading to activation of oxygen molecules due to their interaction with $\mathrm{S}, \mathrm{N}$-doped support. On the other hand, the presence of active oxygen on GQDs surface enhance the aerobic oxidation of organic compounds [64]. Therefore, the enhanced photocatalytic properties of FeTSPP@S, N: GQDs compared to FeTSPP catalyst may be attributed to (I) the increase of quantum yield in S, N: GQDs owning to the high loading of iron porphyrine, (II) $\pi$-electron interaction between the aromatic groups of reactants and $S, N$ : GQDs support, favoring the close proximity of the reactants to the catalytic sites and (III) activation of oxygen molecules by using $\mathrm{S}, \mathrm{N}$ : GQDs as support. In addition, employing $\mathrm{S}, \mathrm{N}$ : GQDs as a catalytic support not only increase the stability of photocatalyst, but also facilitate the recovery process for aerobic oxidation reactions. Moreover, the control experiments with S, N: GQDs, FeTSPP and FeTSPP@S,N: GQDs under dark were carried and the conversions were obtained to be 20,27 , and $45 \%$, respectively. According to the results, the conversions under dark conditions as compared to those of visible light were reduced owning to the lower activated oxygen.

Table. 2 Aerobic oxidation of benzyl alcohol by various photocatalysts

\begin{tabular}{|c|c|c|c|}
\hline Entry & Photocatalyst & Conversion (\%) & Selectivity (\%) \\
\hline 1 & FeTSPP@ S, N: GQDs & 85 & 94 \\
\hline 2 & S, N: GQDs & 33 & 52 \\
\hline 3 & N: GQDs & 26 & 49 \\
\hline 4 & GQDs & 17 & 45 \\
\hline 5 & FeTSPP & 56 & 74 \\
\hline
\end{tabular}


The results for our photocatalyst have been compared with previously reported photocatalysts summarized in Table 3. The as-prepared FeTSPP@S, N: GQDs photocatalyst gives high reaction yield in water in a shorter reaction time at room temperature.

Table. 3 Comparison of the results obtained from FeTSPP@S, N: GQDs with various types of photocatalysts for the aerobic oxidation of benzyl alcohol

\begin{tabular}{|c|c|c|c|c|c|c|c|c|}
\hline Entry & Photocatalyst & Solvent & $\begin{array}{l}\text { Temp } \\
\left({ }^{\circ} \mathrm{C}\right)\end{array}$ & $\begin{array}{l}\text { Time } \\
\text { (h) }\end{array}$ & $\begin{array}{c}\text { Conversion } \\
\text { (\%) }\end{array}$ & $\begin{array}{c}\text { Selectivity } \\
\text { (\%) }\end{array}$ & Light sources & Ref \\
\hline 1 & $\begin{array}{c}\text { FeTSPP@S, N: } \\
\text { GQDs }\end{array}$ & Water & 25 & 14 & 85 & 94 & $\begin{array}{l}\text { Six fluorescent circular } \\
\text { lamp, } 22 \mathrm{~W}, 230 \mathrm{~V}\end{array}$ & $\begin{array}{l}\text { This } \\
\text { work }\end{array}$ \\
\hline 2 & $\mathrm{AR}^{1} / \mathrm{TiO}_{2}{ }^{2} / \mathrm{TEMPO}^{3}$ & Benzotrifluoride & 25 & 18 & 80 & 98 & Halogen lamp, $500 \mathrm{~W}$ & {$[17]$} \\
\hline 3 & Graphene/ $/ \mathrm{TiO}_{2}$ & Benzotrifluoride & 25 & 20 & 62 & 100 & Xe arc lamp, 300W & [83] \\
\hline 4 & $\mathrm{Au} / \mathrm{TiO}_{2}$ & Toluene & 25 & 4 & 85 & 79 & Xe lamp, $2 \mathrm{~kW}$ & [84] \\
\hline 5 & $\mathrm{HCl} / \mathrm{TiO}_{2}$ & Benzotrifluoride & 25 & 4 & 66 & 99 & Hg-lamp, $100 \mathrm{~W}$ & {$[18]$} \\
\hline 6 & $\begin{array}{l}\text { Polyoxometalate- } \\
\text { zirconia }\end{array}$ & Acetonitrile & 25 & 4 & 81 & - & $\begin{array}{c}\text { High-pressure } \\
\text { mercury lamp, } 400 \mathrm{~W}\end{array}$ & {$[85]$} \\
\hline 7 & $\mathrm{mpg}-\mathrm{C}_{3} \mathrm{~N}_{4}{ }^{5}$ & Trifluorotoluene & 100 & 3 & 57 & $>99$ & Xenon lamp, $300 \mathrm{~W}$ & [86] \\
\hline 8 & treated $\mathrm{TiO}_{2}$ & Benzotrifluoride & 25 & 12 & 43 & 99 & $\begin{array}{l}\text { High-pressure Hg lamp, } \\
100 \mathrm{~W}\end{array}$ & [87] \\
\hline 9 & $\mathrm{CdS}-5 \% \mathrm{SEG}^{6}$ & Water & 25 & 4 & 67 & 72 & Xe arc lamp, 300-W & {$[88]$} \\
\hline 10 & $\mathrm{Cu} / \mathrm{Nb}_{2} \mathrm{O}_{5}{ }^{7}$ & Solvent-free & 25 & 24 & 37 & 99 & $\begin{array}{c}\text { High pressure } \\
\text { mercury lamp, } 400 \mathrm{~W}\end{array}$ & [89] \\
\hline 11 & $\mathrm{Pt}^{8} / \mathrm{TiO}_{2}$ & Water & 35 & 2 & 87 & 68 & $\begin{array}{c}\text { High pressure } \\
\text { mercury lamp, } 400 \mathrm{~W}\end{array}$ & {$[90]$} \\
\hline 12 & $\mathrm{Bi}_{2} \mathrm{WO}_{6}$ & Water & 25 & 10 & 58 & 99 & $\begin{array}{c}\text { Ultra-high-pressure } \mathrm{Hg} \\
\text { lamp, } 500 \mathrm{~W}\end{array}$ & [91] \\
\hline 13 & $\mathrm{UiO}-66\left(\mathrm{NH}_{2}\right)$ & Benzotrifluoride & 25 & 4 & 21.2 & $>99$ & $\begin{array}{c}\text { High pressure } \\
\text { mercury lamp, } 400 \mathrm{~W}\end{array}$ & [92] \\
\hline
\end{tabular}




\begin{tabular}{|c|c|c|c|c|c|c|c|c|}
\hline 14 & Pd-Ir/TiO & Solvent-free & 60 & 6 & 25.6 & 82 & Xenon lamp, 400 W & [93] \\
\hline 15 & CdS-UiO-66 $\left(\mathrm{NH}_{2}\right)$ & Benzotrifluoride & 25 & 4 & 30.12 & $>99$ & Medium pressure & [94] \\
\hline
\end{tabular}

${ }^{1}$ Alizarin red; ${ }^{2}$ Titania; ${ }^{3}$ 2,2,6,6-tetramethylpiperidinyloxyl; ${ }^{4}$ Gold nanoparticle; ${ }^{5}$ Mesoporous carbon nitride polymer;

${ }^{6}$ Solvent-exfoliated graphene; ${ }^{7}$ Niobium oxide; ${ }^{8}$ Platinum nanoparticle

\subsection{Stability of FeTSPP@S, N: GQDs photocatalyst}

The recovery of FeTSPP@S, N: GQDs photocatalyst was easy and efficient. The reaction mixture was filtered off after $2 \mathrm{~h}$ and black FeTSPP@S, N: GQDs solid then washed with $\mathrm{CH}_{3} \mathrm{Cl}$ as safe solvent, dried under vacuum, and used directly for the next run of reaction without further purification. The conversion yields and selectivity for every run are presented in Fig. 7a. The ease of recovery combined with the intrinsic stability of S, N: GQDs component, not only allows the catalyst to be recovered efficiently but also lead to high conversion yields and selectivity over at least six times in the aerobic oxidation of benzyl alcohols. Moreover, the productivity of the as-prepared photocatalyst was evaluated for the aerobic oxidation of benzyl alcohol up to five cycles after completion of the reaction. According to the results as shown in Fig. 7b, the conversion yield did not change significantly after every run, which indicates the high productivity of the FeTSPP@S, N: GQDs photocatalyst.

\section{Conclusion}

A novel, efficient, green and recoverable visible-light photocatalyst was synthesized using an anionic FeTSPP supported on S, N: GQDs surface. The selective aerobic oxidation of benzyl alcohols to the related aldehydes were considerably enhanced in the presence of a low amount of FeTSPP@S, N: GQDs photocatalyst loading with excellent selectivity. The attained photocatalytic performance may be attributed to the reduced band gap, leading to broad absorption bands in the visible light region, large surface area, enhanced electronic density, and $\pi-\pi$ interactions between oxidizing agent and reagents on photocatalyst surface. No additives, toxic reactants or solvents were applied and no laborious purification was essential. Moreover, the use of visible and white light and water as a green solvent, as well as mild reaction conditions, easy work-up procedure, and recovery and reusability of photocatalyst lead to a cost 
effective and environmentally friendly photocatalytic methodology which makes it suitable for industrial applications.

\section{Acknowledgments}

The financial support provided by the Iran National Science Foundation (INSF) is gratefully acknowledged.

\section{References}

[1] G.W. Parshall, S.D. Ittel, Homogeneous catalysis, Wiley, 1992.

[2] B. Cornils, W.A. Herrmann, Applied homogeneous catalysis with organometallic compounds, VCH Weinheim etc., 1996.

[3] S.D. Burke, R.L. Danheiser, Oxidizing and reducing agents, Wiley Chichester:, 1999.

[4] G.-J. ten Brink, I.W. Arends, R.A. Sheldon, Science 287 (2000) 1636-1639.

[5] A.B. Sorokin, A. Tuel, Catalysis today 57 (2000) 45-59.

[6] B. Meunier, Chemical Reviews 92 (1992) 1411-1456.

[7] I.A. Ansari, R. Gree, Organic letters 4 (2002) 1507-1509.

[8] B.-Z. Zhan, A. Thompson, Tetrahedron 60 (2004) 2917-2935.

[9] M.J. Schultz, M.S. Sigman, Tetrahedron 62 (2006) 8227-8241.

[10] P.J. Figiel, M. Leskelä, T. Repo, Advanced Synthesis \& Catalysis 349 (2007) 1173-1179.

[11] P.J. Figiel, A. Sibaouih, J.U. Ahmad, M. Nieger, M.T. Räisänen, M. Leskelä, T. Repo, Advanced Synthesis \& Catalysis 351 (2009) 2625-2632.

[12] G. Wu, X. Wang, J. Li, N. Zhao, W. Wei, Y. Sun, Catalysis Today 131 (2008) 402-407.

[13] P. Chaudhuri, M. Hess, J. Müller, K. Hildenbrand, E. Bill, T. Weyhermüller, K. Wieghardt, Journal of the American Chemical Society 121 (1999) 9599-9610.

[14] P. Chaudhuri, M. Hess, T. Weyhermüller, K. Wieghardt, Angewandte Chemie International Edition 38 (1999) 1095-1098.

[15] G. Cainelli, G. Cardillo, Chromium oxidations in organic chemistry, Springer Science \& Business Media, 2012.

[16] M. Hajimohammadi, N. Safari, H. Mofakham, F. Deyhimi, Green Chemistry 13 (2011) 991-997.

[17] M. Zhang, C. Chen, W. Ma, J. Zhao, Angewandte Chemie 120 (2008) 9876-9879.

[18] Q. Wang, M. Zhang, C. Chen, W. Ma, J. Zhao, Angewandte Chemie International Edition 49 (2010) 7976-7979.

[19] M. Rueping, C. Vila, A. Szadkowska, R.M. Koenigs, J. Fronert, ACS Catalysis 2 (2012) 2810-2815.

[20] R.A. Sheldon, Metalloporphyrins in catalytic oxidations, CRC Press, 1994.

[21] T. Mlodnicka, Journal of molecular catalysis 36 (1986) 205-242.

[22] K.M. Smith, J.E. Falk, Porphyrins and metalloporphyrins, Elsevier Amsterdam, 1975.

[23] Z.J. LI, X. Zhen-Qiang, Chinese Journal of Organic Chemistry 1 (2004).

[24] P. Böhm, H. Gröger, ChemCatChem 7 (2015) 22-28.

[25] M. Calvete, G.Y. Yang, M. Hanack, Synthetic Metals 141 (2004) 231-243.

[26] V. Eswaraiah, V. Sankaranarayanan, S. Ramaprabhu, Macromolecular Materials and Engineering 296 (2011) 894-898.

[27] G.S. Machado, K.A.D. de Freitas Castro, F. Wypych, S. Nakagaki, Journal of Molecular Catalysis A: Chemical 283 (2008) 99-107. 
[28] V. Mirkhani, S. Tangestaninejad, M. Moghadam, Z. Karimian, Bioorganic \& medicinal chemistry letters 13 (2003) 3433-3435.

[29] M. Nandi, P. Roy, H. Uyama, A. Bhaumik, Dalton Transactions 40 (2011) 12510-12518.

[30] M. Mahyari, M.S. Laeini, A. Shaabani, Chemical Communications 50 (2014) 7855-7857.

[31] M. Mahyari, A. Shaabani, Applied Catalysis A: General 469 (2014) 524-531.

[32] D. Qu, M. Zheng, P. Du, Y. Zhou, L. Zhang, D. Li, H. Tan, Z. Zhao, Z. Xie, Z. Sun, Nanoscale 5 (2013) 12272-12277.

[33] T.F. Yeh, C.Y. Teng, S.J. Chen, H. Teng, Advanced Materials 26 (2014) 3297-3303.

[34] B.-X. Zhang, H. Gao, X.-L. Li, New Journal of Chemistry 38 (2014) 4615-4621.

[35] Y. Song, S. Chen, ACS applied materials \& interfaces 6 (2014) 14050-14060.

[36] Q. Lu, W. Wei, Z. Zhou, Z. Zhou, Y. Zhang, S. Liu, Analyst 139 (2014) 2404-2410.

[37] L.L. Li, J. Ji, R. Fei, C.Z. Wang, Q. Lu, J.R. Zhang, L.P. Jiang, J.J. Zhu, Advanced Functional Materials 22 (2012) 2971-2979.

[38] D. Pan, J. Zhang, Z. Li, M. Wu, Advanced Materials 22 (2010) 734-738.

[39] L. Zheng, Y. Chi, Y. Dong, J. Lin, B. Wang, Journal of the American Chemical Society 131 (2009) 4564-4565.

[40] Y. Dong, N. Zhou, X. Lin, J. Lin, Y. Chi, G. Chen, Chemistry of Materials 22 (2010) 5895-5899.

[41] Q. Wang, H. Zheng, Y. Long, L. Zhang, M. Gao, W. Bai, Carbon 49 (2011) 3134-3140.

[42] R. Liu, D. Wu, S. Liu, K. Koynov, W. Knoll, Q. Li, Angewandte Chemie 121 (2009) 4668-4671.

[43] Y. Dong, H. Pang, H.B. Yang, C. Guo, J. Shao, Y. Chi, C.M. Li, T. Yu, Angewandte Chemie International Edition 52 (2013) 7800-7804.

[44] A. Shaabani, M. Mahyari, Journal of Materials Chemistry A 1 (2013) 9303-9311.

[45] M. Mahyari, A. Shaabani, Y. Bide, RSC Advances 3 (2013) 22509-22517.

[46] H. Hosseini, M. Mahyari, A. Bagheri, A. Shaabani, Biosensors and Bioelectronics 52 (2014) 136-

142.

[47] H. Hosseini, M. Mahyari, A. Bagheri, A. Shaabani, Journal of Power Sources 247 (2014) 70-77.

[48] M. SadegháLaeini, Chemical Communications 50 (2014) 7855-7857.

[49] M. Mahyari, A. Shaabani, M. Behbahani, A. Bagheri, green chemistry 25 (2014) 27.

[50] M. Mahyari, M.S. Laeini, A. Shaabani, H. Kazerooni, Applied Organometallic Chemistry 29 (2015) 456-461.

[51] Y. Dong, G. Li, N. Zhou, R. Wang, Y. Chi, G. Chen, Analytical chemistry 84 (2012) 8378-8382.

[52] J.N. Gavgani, A. Hasani, M. Nouri, M. Mahyari, A. Salehi, Sensors and Actuators B: Chemical (2016).

[53] L. Zhang, D. Peng, R.-P. Liang, J.-D. Qiu, Analytical chemistry 87 (2015) 10894-10901.

[54] Y. Li, Y. Zhao, H. Cheng, Y. Hu, G. Shi, L. Dai, L. Qu, Journal of the American Chemical Society 134 (2011) 15-18.

[55] K. Gong, F. Du, Z. Xia, M. Durstock, L. Dai, science 323 (2009) 760-764.

[56] S. Wang, D. Yu, L. Dai, Journal of the American Chemical Society 133 (2011) 5182-5185.

[57] P.A. Denis, R. Faccio, A.W. Mombru, ChemPhysChem 10 (2009) 715-722.

[58] C. Pérollier, A.B. Sorokin, Chemical Communications (2002) 1548-1549.

[59] R. Naik, P. Joshi, R.K. Deshpande, Journal of Molecular Catalysis A: Chemical 238 (2005) 46-50.

[60] M. Alvaro, E. Carbonell, M. Esplá, H. Garcia, Applied Catalysis B: Environmental 57 (2005) 37-42.

[61] S.L. Jain, B. Sain, Journal of Molecular Catalysis A: Chemical 176 (2001) 101-104.

[62] Q. Lu, Y. Zhang, S. Liu, Journal of Materials Chemistry A 3 (2015) 8552-8558.

[63] S.-A. Wohlgemuth, R.J. White, M.-G. Willinger, M.-M. Titirici, M. Antonietti, Green Chemistry 14 (2012) 1515-1523.

[64] J. Long, X. Xie, J. Xu, Q. Gu, L. Chen, X. Wang, ACS Catalysis 2 (2012) 622-631. 
[65] M. Li, W. Wu, W. Ren, H.-M. Cheng, N. Tang, W. Zhong, Y. Du, Applied Physics Letters 101 (2012) 103107.

[66] Y. Li, Y. Hu, Y. Zhao, G. Shi, L. Deng, Y. Hou, L. Qu, Advanced Materials 23 (2011) 776-780.

[67] J. Liang, Y. Jiao, M. Jaroniec, S.Z. Qiao, Angewandte Chemie International Edition 51 (2012) 11496-11500.

[68] L. Tang, R. Ji, X. Cao, J. Lin, H. Jiang, X. Li, K.S. Teng, C.M. Luk, S. Zeng, J. Hao, ACS nano 6 (2012) 5102-5110.

[69] S. Zhu, J. Zhang, S. Tang, C. Qiao, L. Wang, H. Wang, X. Liu, B. Li, Y. Li, W. Yu, Advanced Functional Materials 22 (2012) 4732-4740.

[70] D. Sun, R. Ban, P.-H. Zhang, G.-H. Wu, J.-R. Zhang, J.-J. Zhu, Carbon 64 (2013) 424-434.

[71] D. Lin-Vien, N.B. Colthup, W.G. Fateley, J.G. Grasselli, The handbook of infrared and Raman characteristic frequencies of organic molecules, Elsevier, 1991.

[72] G. Lawrie, I. Keen, B. Drew, A. Chandler-Temple, L. Rintoul, P. Fredericks, L. Grøndahl, Biomacromolecules 8 (2007) 2533-2541.

[73] B. Rao, K.C. Mouli, N. Rambabu, A. Dalai, R. Prasad, Catalysis Communications 14 (2011) 20-26.

[74] B. Chang, J. Fu, Y. Tian, X. Dong, RSC Advances 3 (2013) 1987-1994.

[75] M.E. Lipińska, S.L. Rebelo, C. Freire, Journal of Materials Science 49 (2014) 1494-1505.

[76] M. Rutkowska, A. Eisenberg, Macromolecules 17 (1984) 821-824.

[77] M.M. Mahat, D. Mawad, G.W. Nelson, S. Fearn, R.G. Palgrave, D.J. Payne, M.M. Stevens, Journal of Materials Chemistry C 3 (2015) 7180-7186.

[78] T. Yamashita, P. Hayes, Applied Surface Science 254 (2008) 2441-2449.

[79] S. Golczak, A. Kanciurzewska, M. FahIman, K. Langer, J.J. Langer, Solid State Ionics 179 (2008) 2234-2239.

[80] Z. Yang, Z. Yao, G. Li, G. Fang, H. Nie, Z. Liu, X. Zhou, X.a. Chen, S. Huang, ACS nano 6 (2011) 205-

211.

[81] S. Sahu, B. Behera, T.K. Maiti, S. Mohapatra, Chemical Communications 48 (2012) 8835-8837.

[82] M.-S. Park, J.-S. Yu, K.J. Kim, G. Jeong, J.-H. Kim, Y.-N. Jo, U. Hwang, S. Kang, T. Woo, Y.-J. Kim, Physical Chemistry Chemical Physics 14 (2012) 6796-6804.

[83] Y. Zhang, Z.-R. Tang, X. Fu, Y.-J. Xu, ACS nano 5 (2011) 7426-7435.

[84] D. Tsukamoto, Y. Shiraishi, Y. Sugano, S. Ichikawa, S. Tanaka, T. Hirai, Journal of the American Chemical Society 134 (2012) 6309-6315.

[85] S. Farhadi, M. Zaidi, Applied Catalysis A: General 354 (2009) 119-126.

[86] F. Su, S.C. Mathew, G. Lipner, X. Fu, M. Antonietti, S. Blechert, X. Wang, Journal of the American Chemical Society 132 (2010) 16299-16301.

[87] M. Zhang, Q. Wang, C. Chen, L. Zang, W. Ma, J. Zhao, Angewandte Chemie International Edition 48 (2009) 6081-6084.

[88] N. Zhang, M.-Q. Yang, Z.-R. Tang, Y.-J. Xu, Journal of Catalysis 303 (2013) 60-69.

[89] S. Furukawa, A. Tamura, T. Shishido, K. Teramura, T. Tanaka, Applied Catalysis B: Environmental 110 (2011) 216-220.

[90] W. Zhai, S. Xue, A. Zhu, Y. Luo, Y. Tian, ChemCatChem 3 (2011) 127-130.

[91] Y. Zhang, Y.-J. Xu, RSC Advances 4 (2014) 2904-2910.

[92] L. Shen, S. Liang, W. Wu, R. Liang, L. Wu, Dalton Transactions 42 (2013) 13649-13657.

[93] W. Feng, G. Wu, L. Li, N. Guan, Green Chemistry 13 (2011) 3265-3272.

[94] L. Shen, S. Liang, W. Wu, R. Liang, L. Wu, Journal of Materials Chemistry A 1 (2013) 11473-11482. 


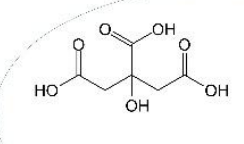

Citric acid

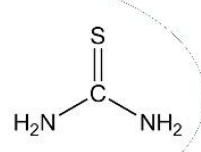

Thiourea
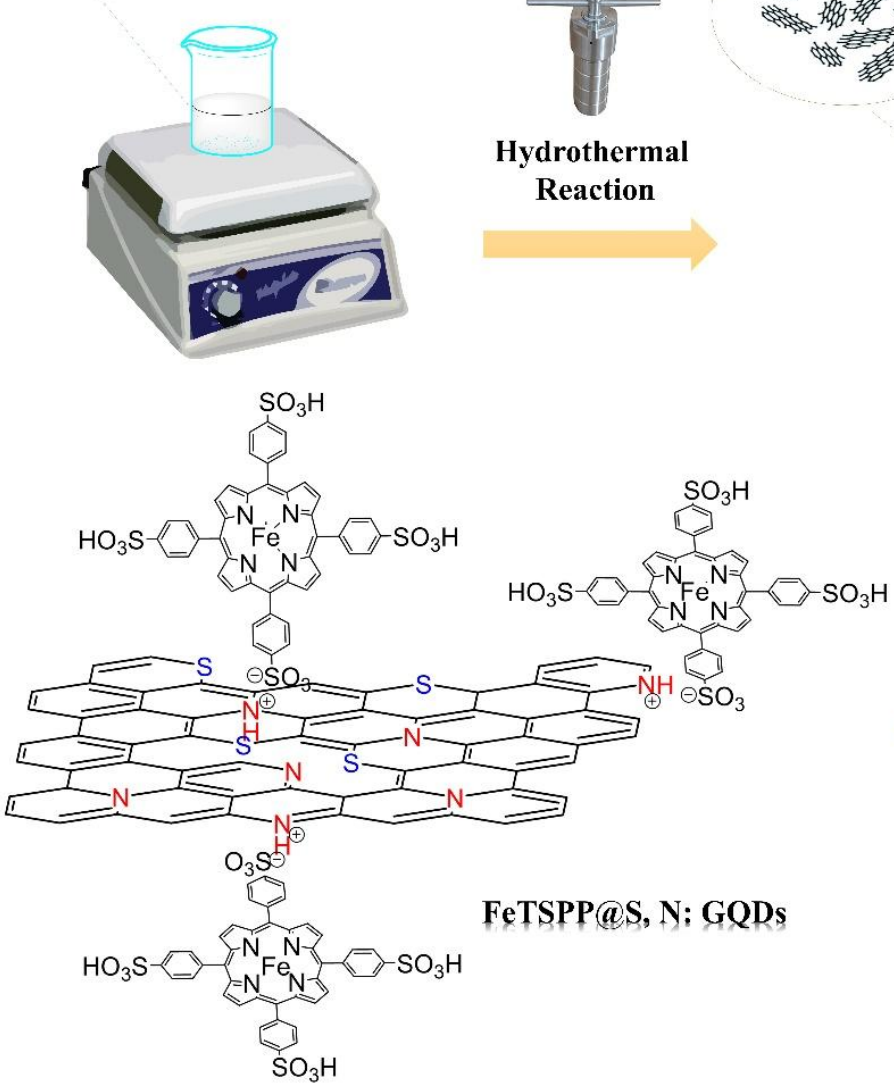

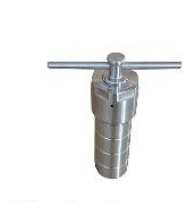

Hydrothermal Reaction

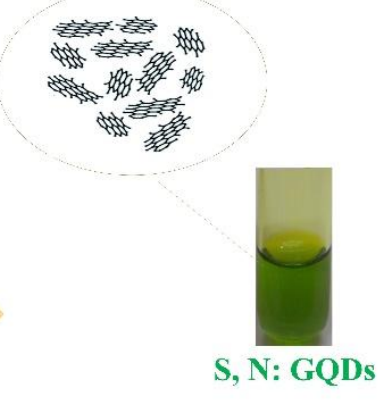

S, N: GQDs

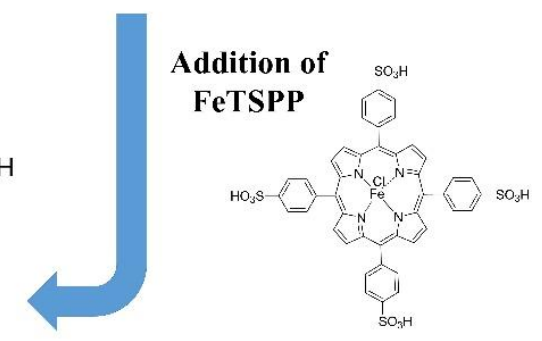

Fig. 1 Synthesis of FeTSPP@S, N: GQDs. 

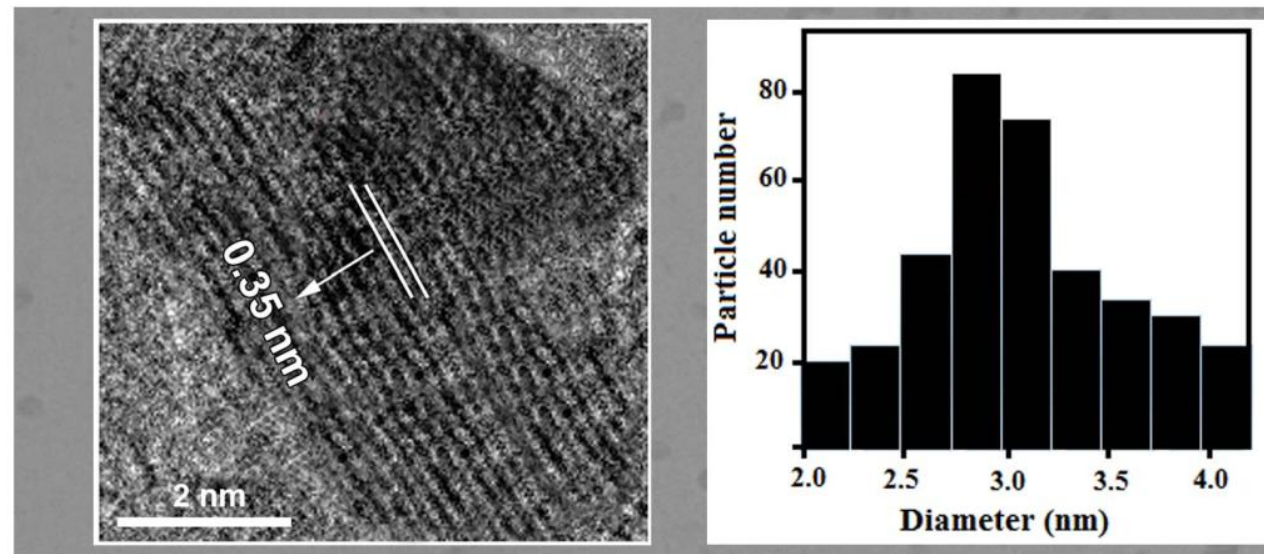

$10 \mathrm{~nm}$

Fig. 2 TEM image of FeTSPP@S, N: GQDs. The corresponding size distribution and HRTEM image are inserted as insets. 


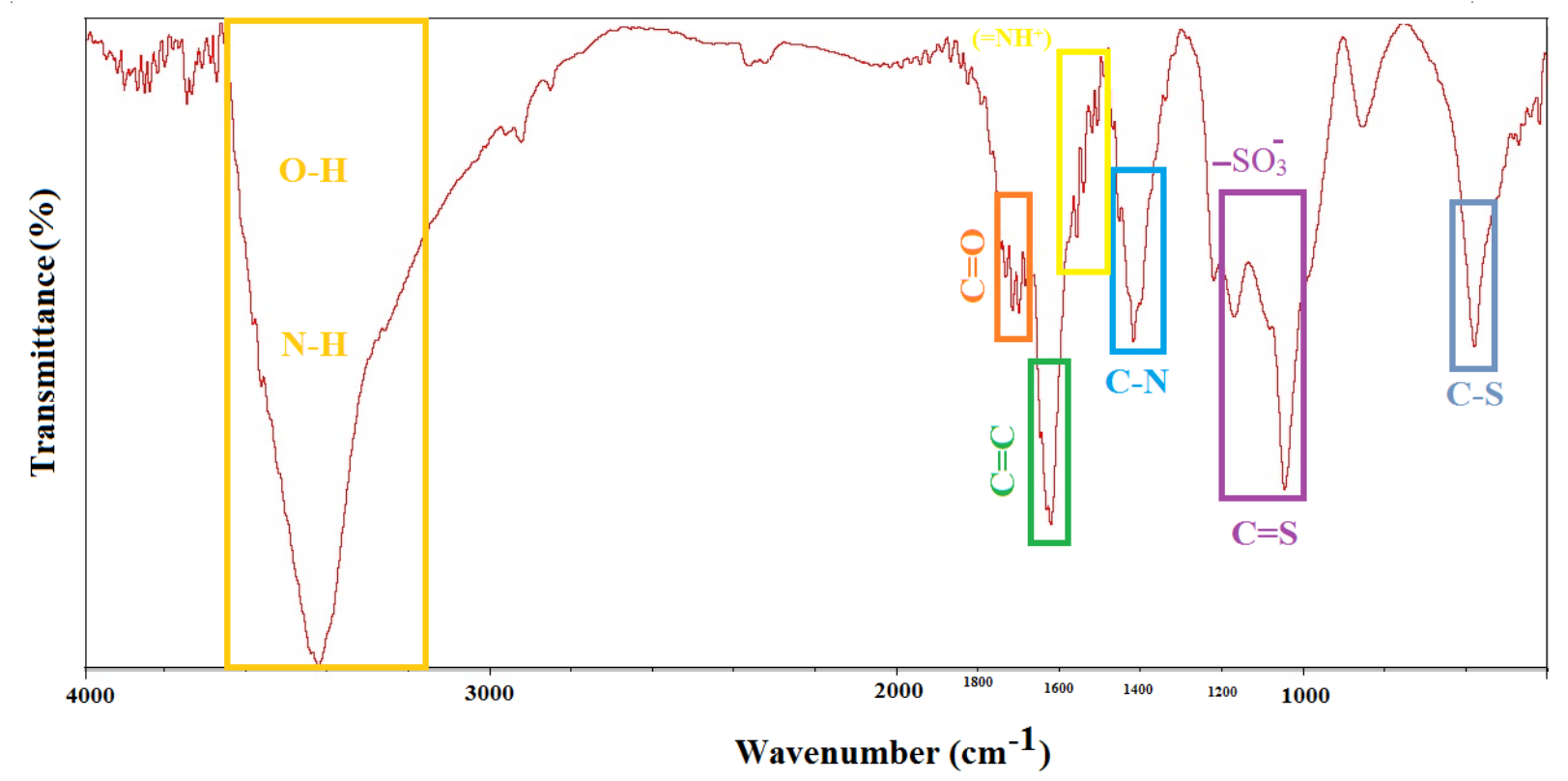

Fig. 3 FTIR spectrum of FeTSPP@S, N: GQDs. 

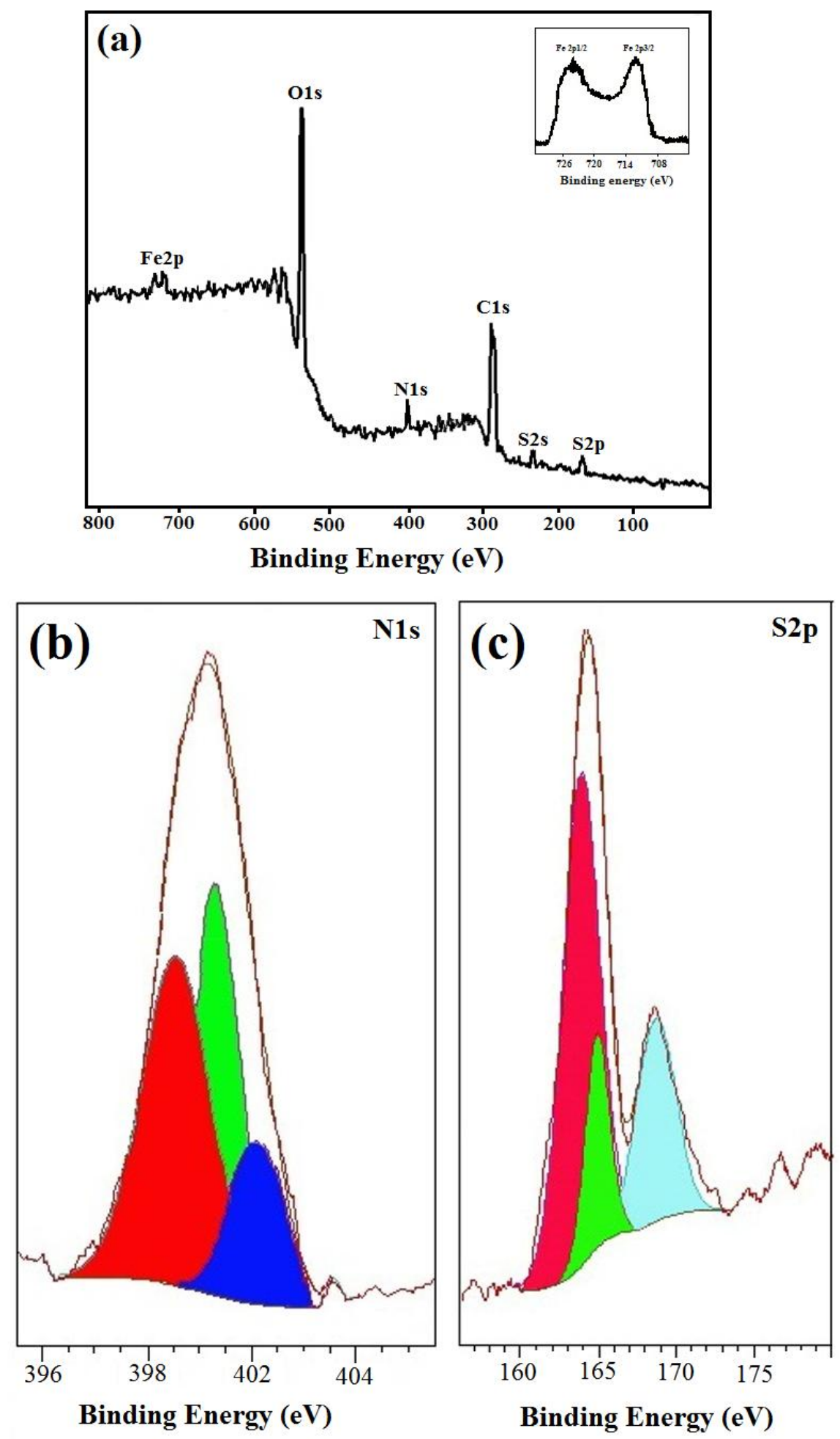

Fig. 4 XPS full survey of FeTSPP@S, N: GQDs (a). The high resolution XPS of N1s (b) and S2p (c) spectra of FeTSPP@S, N: GQDs. 


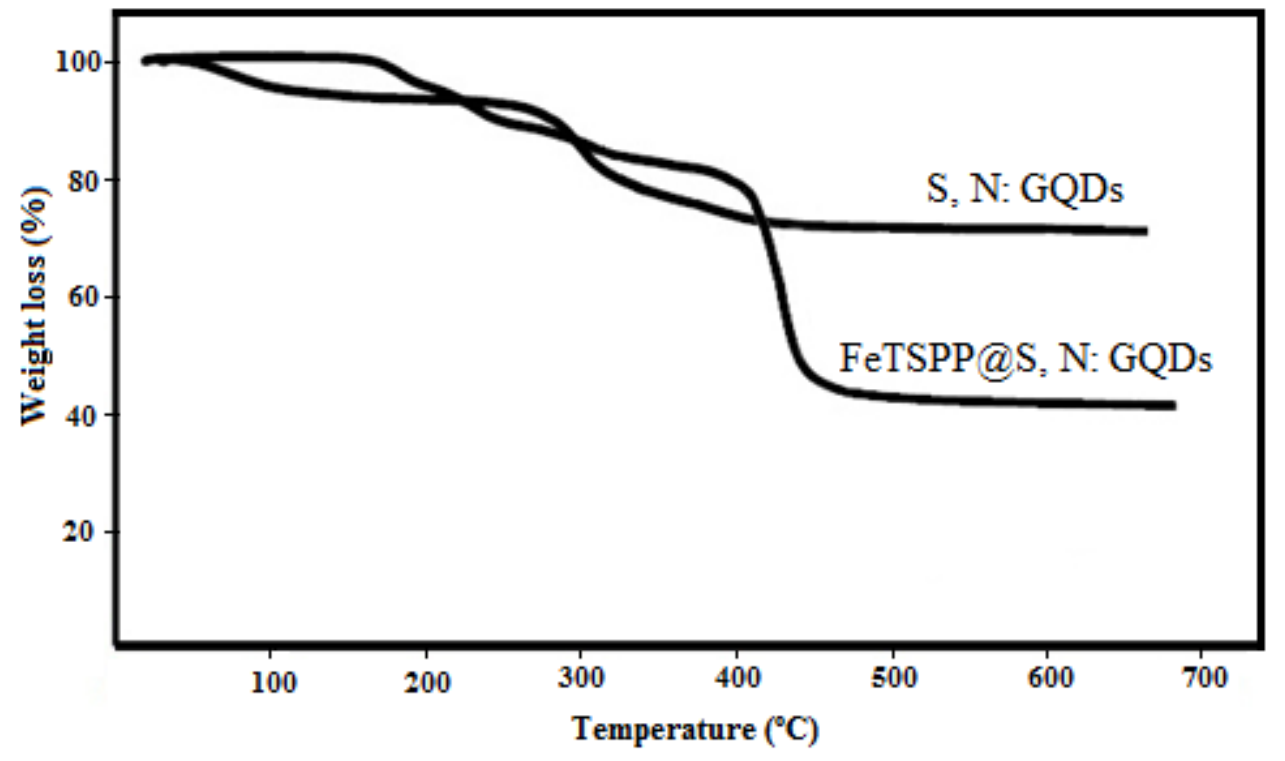

Fig. 5 TGA curves of S, N: GQDs, and FeTSPP@S, N: GQDs. 


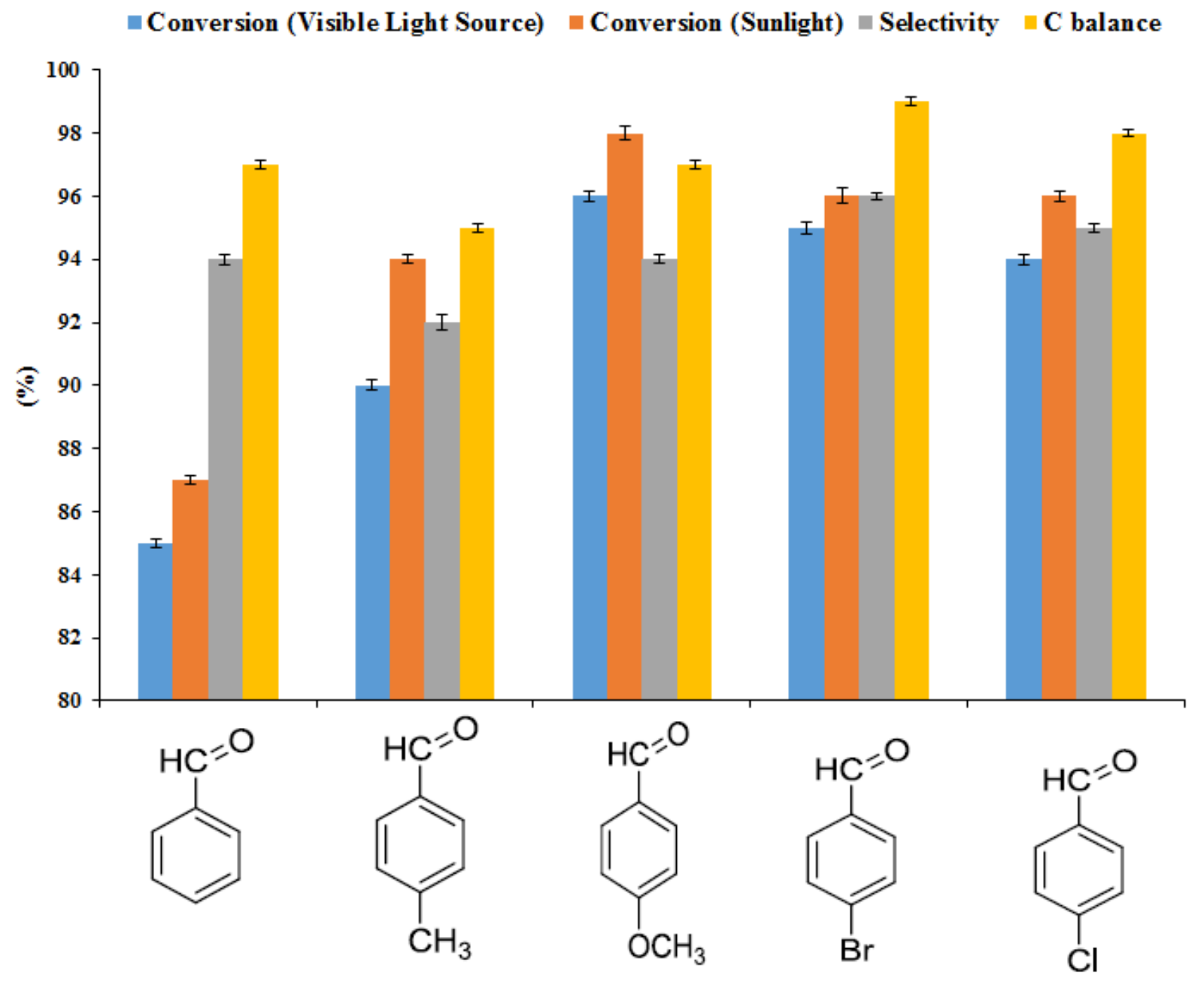

$1.0 \mathrm{~mol}$ of alcohol substrate, catalyst $(1.0 \mathrm{mg})$, air $(1 \mathrm{~atm})$ and $5.0 \mathrm{~mL} \mathrm{\textrm {H } _ { 2 }} \mathrm{O}$ solvent. Visible light source: Fluorescent circular lamp, six 22W lamps (32400 LUX) and Sunlight (62000 LUX).

Fig. 6 Aerobic oxidation of benzyl alcohols using FeTSPP@S, N: GQDs photocatalyst under visible light source and sun light. 


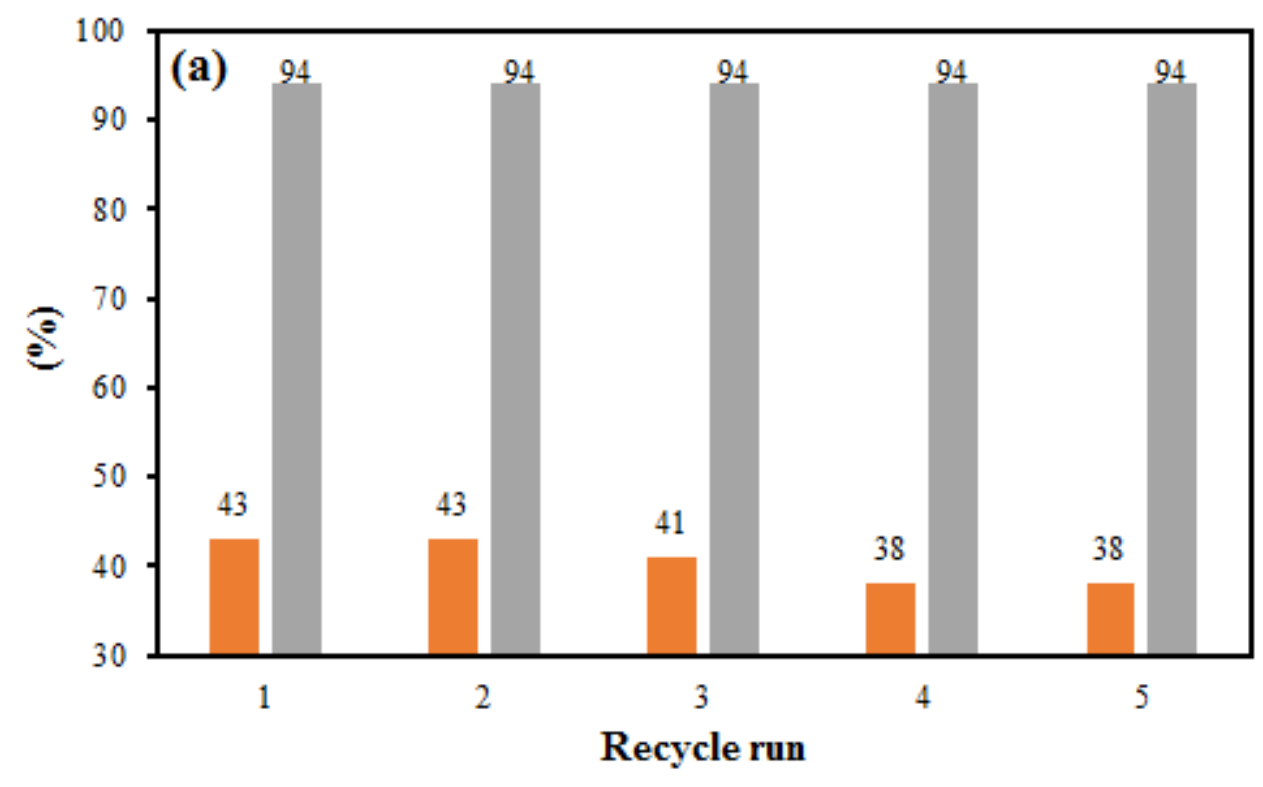

n Conversion

- Selectivity

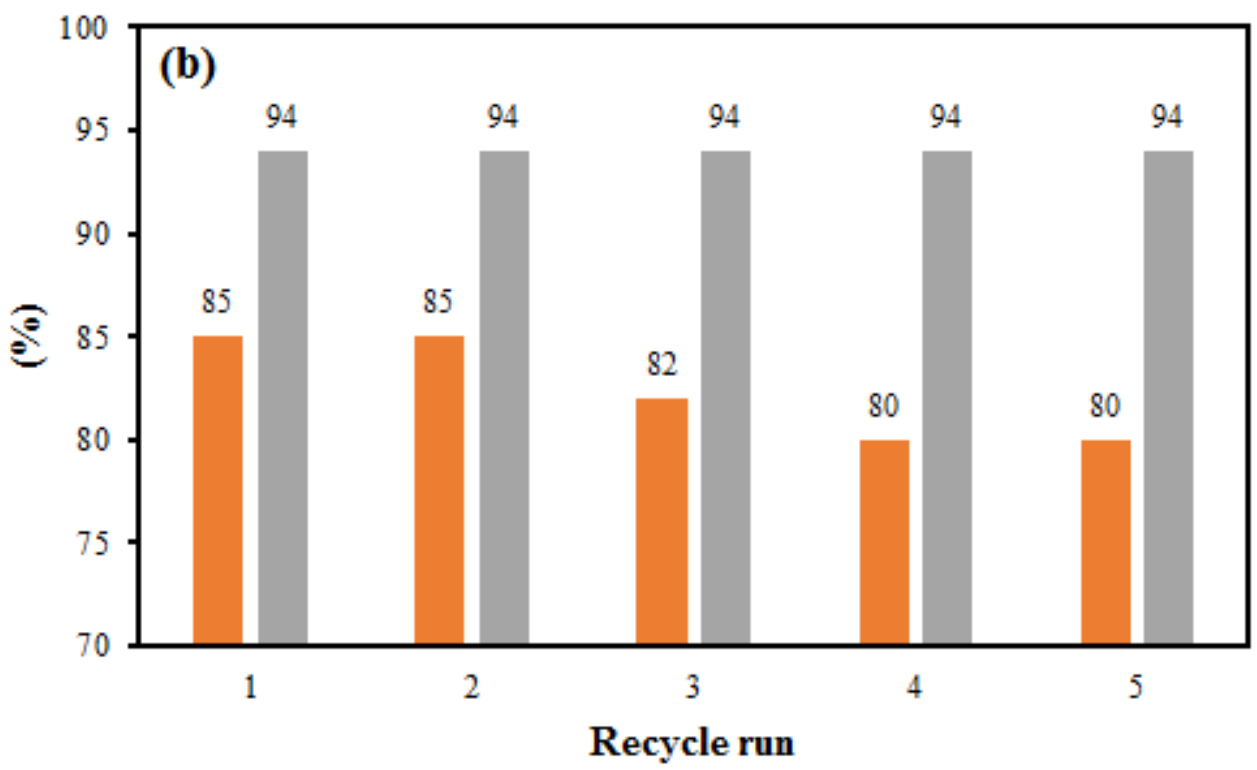

Fig. 7 Recycling of the as-prepared FeTSPP@S, N: GQDs photocatalyst for the aerobic oxidation of benzyl alcohol in water after (a) $2 \mathrm{~h}$ and (b) $14 \mathrm{~h}$; "Reaction conditions: alcohol (1 mol), FeTSPP@S, N: GQDs (0.1 mol\% Fe, $1 \mathrm{mg})$, water $(5 \mathrm{~mL})$, air (1 atm), $25^{\circ} \mathrm{C}$. 

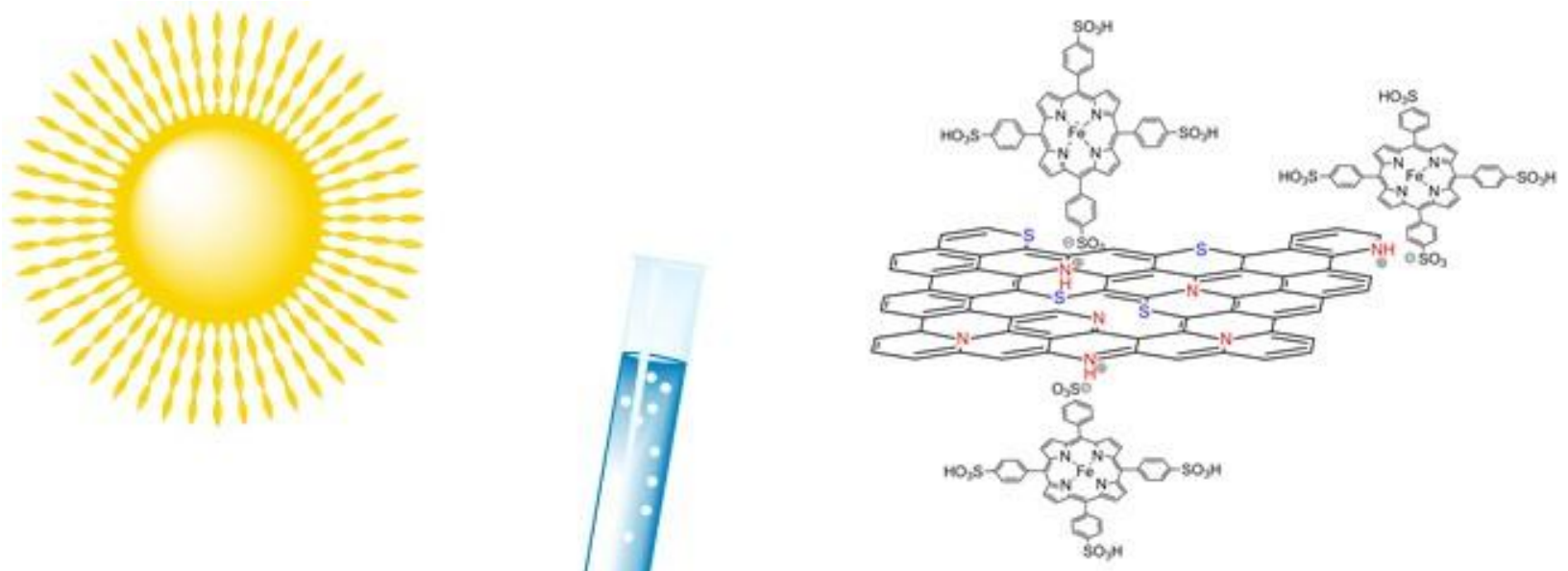

Iron (III) Porphyrin Supported on S and N co-Doped Graphene Quantum Dots

\section{Photocatalytic Aerobic Oxidation}

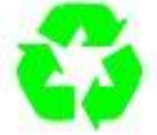

